LA W RENCE LIVERMORE N A TIONAL LABORATORY

\title{
Grain Sorting in Cometary Dust from the Outer Solar Nebula
}

P. J. Wozniakiewicz, J. P. Bradley, H. A. Ishii, D. E. Brownlee, A. T. Kearsley, M. J. Burchell, M. C. Price

October 26, 2012

The Astrophysical Journal Letters 
This document was prepared as an account of work sponsored by an agency of the United States government. Neither the United States government nor Lawrence Livermore National Security, LLC, nor any of their employees makes any warranty, expressed or implied, or assumes any legal liability or responsibility for the accuracy, completeness, or usefulness of any information, apparatus, product, or process disclosed, or represents that its use would not infringe privately owned rights. Reference herein to any specific commercial product, process, or service by trade name, trademark, manufacturer, or otherwise does not necessarily constitute or imply its endorsement, recommendation, or favoring by the United States government or Lawrence Livermore National Security, LLC. The views and opinions of authors expressed herein do not necessarily state or reflect those of the United States government or Lawrence Livermore National Security, LLC, and shall not be used for advertising or product endorsement purposes. 


\title{
Title: Grain sorting in cometary dust from the outer solar nebula
}

\section{Running heading: Sorting in cometary dust}

P. J. Wozniakiewicz ${ }^{1,2} *$, J. P. Bradley ${ }^{1}$, H. A. Ishii ${ }^{1}$, D. E. Brownlee ${ }^{3}$, A. T. Kearsley ${ }^{4}$, M. J. Burchell ${ }^{2}$ M. C. Price ${ }^{2}$

\footnotetext{
${ }^{1}$ Institute of Geophysics and Planetary Physics, Lawrence Livermore National Laboratory, Livermore, CA 94550, USA.

${ }^{2}$ School of Physical Sciences, University of Kent, Canterbury, Kent CT2 7NH, UK.

${ }^{3}$ Department of Astronomy, University of Washington, Seattle WA, USA.

${ }^{4}$ Department of Mineralogy, Natural History Museum, London SW7 5BD, UK.

*To whom correspondence should be addressed. Email: P.Wozniakiewicz@kent.ac.uk
}

\begin{abstract}
Most young stars are surrounded by a disk of gas and dust. Close to the hot stars, amorphous dust grains from the parent molecular cloud are reprocessed into crystals that are then distributed throughout the accretion disk. In some disks, there is a reduction in crystalline grain size with heliocentric distance from the star. We investigated crystalline grain size distributions in chondritic porous $(C P)$ interplanetary
\end{abstract}


dust particles (IDPs) believed to be from small, icy bodies that accreted in outer regions of the solar nebula. The grains are Mg-rich silicates and Fe-rich sulfides, the two most abundant minerals in CP IDPs. We find that they are predominantly $<0.25 \mu \mathrm{m}$ in radius with a mean grain size that varies from one CP IDP to another. We report a size-density relationship between the silicates and sulfides. A similar size-density relationship between much larger silicate and sulfide grains in meteorites from the asteroid belt is ascribed to aerodynamic sorting. Since the silicate and sulfide grains in CP IDPs are theoretically too small for aerodynamic sorting, their size-density relationship may be due to another process capable of sorting small grains.

Key words: accretion, accretion disks - comets: general - Kuiper belt; general protoplanetary disk

\section{INTRODUCTION}

The gas and dust in accretion disks around young stars are leftovers from the interstellar medium (ISM) and the disk formation process itself. Since amorphous silicates are the dominant dust grain component in the ISM ( $>97 \%$, Kemper et al. 2004), the abundant crystalline silicates, observed at mid-infrared wavelengths at the surfaces of protoplanetary disks, must have formed within the disks by reprocessing of amorphous 
ISM silicates (van Boekel et al. 2004; Olofsson et al. 2009). Formation of crystals requires high temperatures believed to have been restricted to the hot inner regions of the accretion disk. In some disks, crystalline silicates are confined to the inner regions, and in others they are redistributed to outer disk regions (van Boekel et al. 2004; Olofsson et al. 2009). In the Solar System, chondritic asteroids and comets, which are generally believed to have accreted between 2 and $4 \mathrm{AU}$ and beyond $5 \mathrm{AU}$ respectively, are considered to have undergone the least parent body processing since accretion and therefore provide an opportunity to study the redistribution of Solar nebula materials at different heliocentric distances (Krot et al. 2003; Brownlee 2003). Refractory calcium aluminum inclusions (CAIs) that formed close to the early Sun are preserved in chondritic meteorites from the asteroid belt, in a sample of Comet 81P/Wild 2 from the inner Kuiper Belt (KB) and in some CP IDPs (MacPherson 2003; Brownlee et al. 2006; Zolensky et al. 2006; Christoffersen \& Buseck 1986; Zolensky 1987; McKeegan 1987). Millimeter-sized grains of refractory silicates are found in meteorites while cometary samples of Comet 81P/Wild 2 and chondritic porous interplanetary dust particles (CP IDPs) contain micron and sub-micron grain sizes (Scott \& Krot 2003; Brownlee et al. 2006; Zolensky et al. 2006; Nakamura et al. 2008; Bradley et al 1988, 1989), consistent with grain size reduction with increasing heliocentric distance observed in disks around some other young stars (van Boekel et al. 2004).

Despite their micrometer-scale dimensions and nanogram masses, CP IDPs are an important class of extraterrestrial materials because they sample parent bodies that are not 
represented among known classes of meteorites. Their porous, fragile structures are consistent with the inferred properties of cometary meteors (Verniani 1969) and their infrared $\sim 10 \mu \mathrm{m}$ silicate features are similar to those of comets (Sandford \& Walker 1985; Bradley et al. 1999; Molster \& Waters 2003). CP IDPs contain at least 10 times more isotopically anomalous presolar constituents than other known meteoritic materials (Nguyen et al. 2007; Messenger 2000). They are composed mostly of amorphous silicates known as GEMS (glass embedded with metals and sulfides), some with isotopic compositions establishing that they are surviving interstellar amorphous silicates (Floss et al. 2006). CP IDPs are the only known class of extraterrestrial materials that do not exhibit evidence of significant post-accretional parent body alteration (Bradley \& Brownlee 1986; Bradley 2003).

Even among the CP IDPs, it has long been recognized that there are differences in average grain size from one IDP to another, but until now, these differences have not been systematically quantified (Fraundorf 1981; Sandford \& Walker 1985; Bradley et al. 1988, 1989). CP IDPs are believed to be from Kuiper Belt (KB) comets like 81P/Wild 2, but variations in mineralogy and abundances of presolar material suggest they are from objects that accreted over a range of heliocentric distances in the KB. We compared the sizes of the most abundant crystalline silicate minerals, low-Fe enstatite $\left(\sim \mathrm{MgSiO}_{3}\right)$ and forsterite $\left(\sim \mathrm{Mg}_{2} \mathrm{SiO}_{4}\right)$, and the sulfide mineral pyrrhotite $\sim \mathrm{Fe}(\mathrm{Ni}) \mathrm{S}$ (with $\mathrm{Ni}<10$ at $\%$ ) in three CP IDPs and Comet 81P/Wild 2 (Figure 1). The 81P/Wild 2 grains are the only available sample from a known KB comet parent body (Brownlee et al. 2006; Zolensky 
et al. 2006). We report that in addition to differences in grain size distributions among and between the CP IDPs and 81P/Wild 2, there is an equivalence in the size-density products of crystalline silicate and sulfide grains suggestive of grain sorting.

\section{GRAIN SIZE DATA COLLECTION AND ANALYSIS}

\subsection{CP IDP specimen preparation and data collection}

The CP IDPs analyzed in this study were U211B6, U220GCA and U212A34A. These were disaggregated into their individual component grains that were then dispersed onto thin carbon support film substrates on copper mesh transmission electron microscope (TEM) grids. Due to their fragile, loosely aggregated structures, the constituent grains readily separate without significant fragmentation (Brownlee \& Joswiak, 2004).

An 80-300 keV FEI TITAN ${ }^{3}$ G2 scanning transmission electron microscope (STEM) was used to measure the CP IDPs. The instrument is equipped with a monochromator, dual spherical aberration $\left(\mathrm{C}_{\mathrm{s}}\right)$ correctors for focused probe and imaging modes, an EDAX Genesis $4000 \mathrm{Si}(\mathrm{Li})$ solid state energy dispersive x-ray spectrometer, and a Gatan Tridiem GIF (Gatan Imaging Filter). High angle annular dark field (HAADF) images were acquired at $300 \mathrm{keV}$ at a magnification of $28500 \mathrm{X}$ from an area totaling approximately $3,000 \mu \mathrm{m}^{2}$ on the U211B6 grid, $1,000 \mu \mathrm{m}^{2}$ on the U220GCA grid and 
$3,000 \mu \mathrm{m}^{2}$ on the U212A34A grid. The images were imported into Adobe Illustrator (Adobe Systems Inc.) where individual grain outlines were traced. Using the ImageJ software package (http://rsbweb.nih.gov/ij/, National Institutes of Health), cross-sectional areas of the outlined grains were measured and grain radii were calculated assuming spherical crystals, a reasonable approximation since the majority of grains are equiaxed. The HAADF images were acquired from overlapping fields-of-view to ensure that grains intersecting the edge of one image were fully exposed in an adjacent image such that their radii could be calculated. Because energy dispersive x-ray spectroscopy (EDX) analyses of over 3000 crystal grains was not experimentally feasible, grain mineralogies were determined based on HAADF contrast and confirmed by compositional measurements using EDX of $>15 \%$ of the population selected at random.

\subsection{CP IDP grain size statistics}

Kolmogorov-Smirnov (K-S) tests comparing the CP IDP datasets indicate the silicate and sulfide grain populations are statistically different both within and between the three particles. To quantify this difference, we determined the mean and standard deviations of each dataset. The size distribution profiles in the CP IDPs are skewed towards smaller sizes and, from K-S tests comparing the datasets against cumulative distribution functions of possible fits (e.g. normal, log-normal, exponential), they are best described by lognormal distributions. Therefore, we use the geometric mean and standard deviation. Since the data were binned in order to extrapolate the fractions of mineral types in each bin (see 
above), calculation of these means and standard deviations directly via analytical expressions was not possible. The mean radii $\left(M_{r}\right)$ and standard deviation $(+\sigma$ and $-\sigma)$ were therefore determined graphically from cumulative frequency distributions as a function of $\ln (r)$, via the modified geometric Folk \& Ward (1957) equations of Blott \& Pye (2001) $)^{1}$ :

$$
\begin{gathered}
M_{r}=\exp \left[\frac{\ln \mathrm{P}_{16}+\ln \mathrm{P}_{50}+\ln \mathrm{P}_{84}}{3}\right] \\
+\sigma=\exp \left[\ln M_{r}+\left(\frac{\ln \mathrm{P}_{16}-\ln \mathrm{P}_{84}}{4}+\frac{\ln P_{5}-\ln \mathrm{P}_{95}}{6.6}\right)\right]-M_{r} \\
-\sigma=M_{r}-\exp \left[\ln M_{r}-\left(\frac{\operatorname{lnP}_{16}-\operatorname{lnP}_{84}}{4}+\frac{\operatorname{lnP}_{5}-\ln \mathrm{P}_{95}}{6.6}\right)\right]
\end{gathered}
$$

Here $\mathrm{P}_{5}, \mathrm{P}_{16}, \mathrm{P}_{50}, \mathrm{P}_{84}$ and $\mathrm{P}_{95}$, are the grain sizes at the $5^{\text {th }}, 16^{\text {th }}, 50^{\text {th }}, 84^{\text {th }}$ and $95^{\text {th }}$ percentile respectively ${ }^{2}$.

Sources of error in individual measurements of radius include errors in identifying and outlining separate grains and the assumption that grains are spherical when converting

1 The graphical method is commonly used in the study of terrestrial sediment grain sizes with similarly skewed distributions and has also been applied previously to extraterrestrial samples (c.f. chondrule measurements by Kuebler et al. 1999). Blott and Pye (2001) derive the standard deviation in ln units. Equations (2) and (3) have been derived from this equation to return the standard deviation in $\mathrm{nm}$ units. 
areas to radii. Error due to the assumption of grain sphericity is minimal because most grains are approximately equi-dimensional and the populations are large. We estimate that the total error in individual radius values based on measurements of area is $\leq 5 \%$. The individual measurement errors are significantly smaller than the calculated geometric standard deviations in $r$, and therefore we are confident that the size distributions obtained accurately represent the size distributions in the CP IDPs. The standard error of the mean of $r$ is the calculated geometric standard deviation divided by the square root of the number of grains measured for each component.

\subsection{Comet $81 \mathrm{P} / \mathrm{Wild} 2$ data collection}

Particle size data for Comet 81P/Wild 2 were taken from a systematic survey of impact craters in Stardust mission Al foil collector surfaces (Price et al. 2010). Craters provide the most reliable means of determining original particle sizes in Comet 81P/Wild 2 due to the well-constrained relationship between original impactor diameter and resulting crater diameter established for the Al foil surfaces by laboratory light gas gun calibration experiments (Kearsley et al. 2006, 2007; Price et al. 2010). (In contrast, particles that impacted the aerogel cells suffered extensive ablation and fragmentation).

During their investigation of grain sizes, Price et al. (2010) made a significant assumption: Where $\mathrm{Mg}, \mathrm{Si}, \mathrm{S}$ and $\mathrm{Fe}$ were detected together in a crater, the impactor was assumed to be composed of two components and crater diameters were divided into two 
equal halves and a particle size calculated for each. This is an oversimplification since analyses of particles in neighboring aerogel collectors indicate these particles are actually aggregates composed of several grains with a range of sizes. For this reason, only data for craters where the chemistry is indicative of single mineral impactors (silicate or sulfide) were included when determining values of $r$ for the comparisons of silicate and sulfide grains in this work.

\subsection{Statistics of the Comet $81 \mathrm{P} /$ Wild2 data}

The geometric mean radii $(r)$ and standard deviation of Comet 81P/Wild 2 silicate and sulfide grains were derived from the data of Price et al. (2010) in the same manner as for the CP IDPs U211B6, U220GCA and U212A34A. Sources of error in the individual measurements of radius (of single component impactors) include the errors in measuring crater diameters and errors in the relationships used to convert crater diameters to impactor diameters. We estimate that the total error in individual radius measurements is $\leq 15 \%$. The individual measurement errors are significantly smaller than the geometric standard deviations in $r$ and are therefore neglected. The standard error of the mean of $r$ is obtained in the same manner as for the CP IDPs. For a full discussion of the biases and simplifications for this dataset, see Price et al. (2010). 


\section{RESULTS}

Cumulative frequency distributions of silicate and sulfide grain sizes in the CP IDPs and Comet 81P/Wild 2 are shown in Figure 2, with solid lines marking geometric mean radii. We observe that the geometric mean radii of the grains vary between the four samples, and note that the silicates and sulfides from Comet 81P/Wild 2 are significantly larger than those in the CP IDPs (this confirms one of the unexpected first observations of the preliminary examination phase of the Comet 81P/Wild 2 sample analysis (see Zolensky et al. 2006)). We also observe that the geometric mean radii of silicates and sulfides within each CP IDP and Comet 81P/Wild 2 are offset from one another, with sulfides being consistently smaller than silicates. Standard deviations about the means are indicated by shaded regions around each cumulative curve in Figure 2. These deviations about the means do not represent measurement error but, instead, reflect the actual range of the grain size. ${ }^{2}$ The observed differences between the means are statistically significant (see section 2.2) so that, although individual grains cannot be assigned to a specific sample based on size, each sample is distinguishable by the mean radii of its silicate and sulfide populations. The measured grain sizes in U220GCA, U211B6 and U212A34A are consistent with size ranges estimated in other CP IDPs (Fraundorf 1981; Bradley 2003; Brownlee \& Joswiak 2004).

\footnotetext{
2 The larger deviations about the mean $\mathrm{r}$ for comet 81P/Wild 2 are the direct result of poor statistics due to the limited population of single mineral Mg-silicate and Fe-sulfide impacts identified to date (Price et al. 2010).
} 
The geometric mean radii of the silicates are plotted against the geometric mean radii of the sulfides for each sample in Figure 3. Here, error bars represent the standard error on the mean and an error-weighted trendline has been fit that satisfies the equation:

$$
\mathrm{M}_{\mathrm{r}} \text { sulfide }=0.62 \mathrm{M}_{\mathrm{r}} \text { silicate }
$$

The slope of this trend line is approximately equal to the ratio of the average silicate to sulfide densities: In CP IDPs, the most common silicates observed are low-Fe ( $\mathrm{Fe} \leq 5$ atomic \%) enstatite $\left(\mathrm{MgSiO}_{3}\right)$ and forsterite, $\left(\mathrm{Mg}_{2} \mathrm{SiO}_{4}\right)$ and sulfides are predominantly pyrrhotite (Bradley 2003), consistent with our TEM EDX measurements of U211B6, U220GCA and U212A34A. Similar mineralogy is reported for Comet 81P/Wild 2 from TEM EDX compositional data published in Zolensky et al. (2006). Therefore, assuming densities of $3.22 \mathrm{~g} \mathrm{~cm}^{-3}$ for the silicates (mid-point of forsterite and enstatite densities, Deer et al. 1992) and $4.71 \mathrm{~g} \mathrm{~cm}^{-3}$ for the sulfides (mid-point of range of possible Ni-Free pyrrhotite densities, Deer et al. 1992), the ratio of silicate to sulfide densities is approximately 0.68 (within $10 \%$ of the observed weighted trend). The geometric mean radii, standard deviation and standard error on the mean are tabulated in Table 1. Thus, the product of the radius and density of the grains are approximately equivalent between silicates and sulfides in all of these cometary samples. 


\section{DISCUSSION}

The statistically-significant differences we observe in grain size distributions in the CP IDPs and Comet 81P/Wild 2, and the equivalence in their mean silicate and sulfide sizedensity products, are consistent with grain sorting. We note that rare kamacite metal grains, with a density $\left(7.91 \mathrm{~g} \mathrm{~cm}^{-3}\right.$; Henderson \& Perry 1954) much higher than those of the silicates and sulfides, also demonstrate size-density equivalence with silicate or sulfide grains, but they are not included in the present dataset due to large uncertainties from the small population ( $<30$ grains) measured to date. At present, it is unknown what physical mechanism is responsible for the grain sorting in these cometary samples.

In chondritic meteorites that accreted in the asteroid belt between 2 and $4 \mathrm{AU}$, there is strong evidence of sorting of silicates, sulfide and metal grains with grain sizes typically several orders of magnitude larger than those in CP IDPs. Narrow size distributions (of comparable width to those reported here) for chondrules (e.g. Hughes 1978; King \& King 1978; Rubin \& Keil 1984; Rubin \& Grossman 1987) and metal/sulfide grains are reported, the latter shifted towards smaller sizes relative to their accompanying chondrules (Dodd 1976; Skinner \& Leenhouts 1993; Kuebler et al. 1999). Aerodynamic sorting of these meteorite components is generally accepted because grains that were aerodynamically equivalent have the same mean size-density product and would have been sorted together prior to accretion (Kuebler et al. 1999). For comparison, the ratio of size-density products between silicates and sulfides in meteorite data range from 1:1.38 
to 1.33:1 (analyzing the raw data of Kuebler et al. 1999), and the CP IDPs and 81P/Wild 2 exhibit ratios ranging from $1: 1.08$ to $1.14: 1$, even closer to a $1: 1$ equivalence.

Several mechanisms of aerodynamic sorting of the large grains in chondritic meteorites have been proposed. Clayton (1980) suggested that sorting may have occurred as a result of gas dynamics: particles penetrate gas following a shockwave to different depths, are deposited from a turbulent gas at different turbulence strengths or simply sediment from a gas at different speeds dependent on their size and density (and therefore coupling to the gas). Alternatively, turbulence could act to concentrate grains of a specific size and density in low-vorticity zones (Cuzzi et al. 2008). These grains could then be accreted to asteroids, preserving this sorting in the chondritic meteorites that sample them. It is unlikely, however, that the grains in CP IDPs or Comet 81P/Wild 2 were sorted by these mechanisms in the inner nebula prior to being transported out to the comet forming regions since grains throughout the size range observed in our CP IDPs would have remained extremely well coupled to the gas and thus well mixed under inner nebula conditions (exhibiting a net motion, or Brownian motion depending on the motion of the gas). It is possible that similar sorting mechanisms may have operated in the cometforming region with reduced gas density and therefore less efficient gas coupling. However, very little is known about the conditions that existed in the early outer nebula, and it is unclear whether these aerodynamic processes could have operated effectively on such small grain sizes. Another mechanism is aerodynamic sorting accompanying grain settling onto the disk after ballistic transport above the plane of the solar accretion disk in 
bipolar outflows (a mechanism of transport proposed by Shu et al. 1997). Smaller and/or less dense grains settle at larger heliocentric distances (Liffman; 2005). However, these models have only been proposed for large grains like those in chondritic meteorites and heliocentric distances out to $3 \mathrm{AU}$. Other proposed large scale mechanisms of transport that may have resulted in aerodynamic sorting include radiation pressure, photophoresis, turbulent diffusion and large-scale circulation currents, transient spiral density waves and advection as a result of viscous expansion of the disk (Vinković 2009; Mousis et al. 2007; Keller et al. 2004; Boss 2008; Jacquet et al. 2011). None of these mechanisms have been applied to sorting of sub micrometer-sized grains like those in CP IDPs.

Sorting of the small grain sizes in the CP IDPs and Comet 81P/Wild 2 by aerodynamic coupling is challenging to reconcile with models, but if the size-density equivalence relationship we observe is either a direct or indirect consequence of sorting during grain transport, then the silicate versus sulfide size plot in Figure 3 may be related to relative parent body accretion distances from the Sun, with smaller $r$ values corresponding to accretion at larger distances. There is evidence consistent with this hypothesis: CP IDPs with the smallest mean grain size also typically contain the highest abundances of amorphous silicates, organic carbonaceous material and circumstellar "stardust" grains from other stars, all of which are expected to be preserved in highest abundances in small bodies in the outermost (coldest) regions of the solar nebula (Bradley 2003; Nguyen et al. 2007; Wirick et al. 2009). 
Although the physical mechanism responsible for the sorting of such small grains is uncertain, we have demonstrated that there is size-density sorting of the silicate and sulfide components of cometary CP IDPs and Comet 81P/Wild 2 samples that accreted in the outer solar nebula. Future nebula models that consider small grains, together with spatially resolved observations, at infrared and millimeter wavelengths, of grain sizes at the surfaces and within the interiors of accretion disks may provide insight into the sorting and transport of small grains around young stars.

Acknowledgements: We thank F. Ciesla, B. Draine, A. N. Krot, A. Needham, M. Sitko, $\mathrm{X}$. Tielens and J. Cuzzi for useful discussions during manuscript preparation. We thank K. Kuebler for providing us with the original meteoritic datasets of Keubler et al. 1999. We also thank the Editor and an anonymous reviewer for their extremely useful comments and suggestions. This work was funded in part by a grant from NASA's Cosmochemistry program (JPB) and LDRD grant 09-ERI-004 (JPB). H.A. Ishii was supported by NASA's Laboratory Analysis of Returned Samples program. Portions of this work were performed under the auspices of the U.S. Department of Energy by LLNL under contract DE-AC52-07NA27344. 


\section{REFERENCES}

Blott, S. J. \& Pye, K. 2001, Earth Surf. Process. Landforms, 26, 1237

van Boekel, R., Min, M., Leinert Ch., et al. 2004, Nature, 432, 479

Boss, A.P. 2008, EPSL, 268, 102

Bradley, J. P. \& Brownlee, D. E. 1986, Science, 231, 1542

Bradley, J. P. 1988, GCA, 52, 889

Bradley, J. P. Germani M. S. \& Brownlee, D. E. 1989, EPSL, 93, 1

Bradley, J. P., Keller, L. P., Snow, T. P., et al. 1999, Science, 285, 1716

Bradley, J. P. 2003, in Meteorites, Comets and Planets, ed. Davis, A. M., Vol. 1 Treatise on Geochemistry eds. Holland, H. D. \& Turekian, K. K. (Elsevier-Perghamon, Oxford), 689

Brownlee, D. E. in Meteorites, Comets and Planets, ed. Davis, A. M., Vol. 1 Treatise on Geochemistry, eds. Holland, H. D. \& Turekian, K. K. (Elsevier-Perghamon, Oxford) 663 
Brownlee, D. E. \& Joswiak, D. J. 2004, Lunar Planet. Sci. Conference XXXV, 1944 (abstr.)

Brownlee, D. E., Tsou, P., Aléon, J., et al. 2006, Science, 314, 1711

Christoffersen, R. \& Buseck, P. R. 1986, Science, 234, 590

Clayton, D. D. 1980, EPSL, 47, 199

Cuzzi, J. N., Hogan, R. C. \& Shariff, K. 2008, ApJ, 687, 1432

Deer, W. A., Howie, R. A. \& Zussman, J. 1992, in An introduction to the rock-forming minerals (Pearson Education Limited, Harlow), 696

Dodd, R. T. 1976, EPSL, 30, 281

Floss, C., Stadermann, F. J., Bradley, J. P. et al. 2006, GCA 70, 2371

Folk, R. L. \& Ward, W. C. 1957, J. Sed. Pet., 27, 3

Fraundorf, P. 1981, GCA, 45, 915 
Hughes, D. W. 1978, EPSL, 38, 391

Henderson, E. P. \& Perry, S. H. 1954, GCA, 6, 221

Jacquet, E., Fromang, S. \& Gounelle, M. 2011, A\&A 526, L8

Kearsley, A. T., Burchell, M. J., Hörz, F., Cole, M. J. \& Schwandt, C. S. 2006, MAPS, 41,167

Kearsley, A. T., Graham, G. A., Burchell, M. J., et al. 2007, MAPS, 42, 191

Keller, Ch., \& Gail, H.-P. 2004, A\&A, 415, 1177

Kemper, K., Vriend, W. J. \& Tielens, A. G. G. M. 2004, ApJ, 609, 826

King, T. V. V. \& King, E. A. 1978, Meteoritics, 13, 47

Krot, A. N., Keil, K., Goodrich, C. A., Scott, E. R. D. \& Weisberg, M. K. 2003, in Meteorites, Comets and Planets, ed. Davis, A. M., Vol. 1 Treatise on Geochemistry, eds. Holland, H. D. \& Turekian, K. K. (Elsevier-Perghamon, Oxford) 83 
Kuebler, K.E., McSween, Jr., H.Y., Carlson, W.D. \& Hirsch, D. 1999, Icarus, 141, 96

Liffman, K. 2005, MAPS, 40, 123

MacPherson, G. J. 2003, in Meteorites, Comets and Planets, ed. Davis, A. M., Vol. 1 Treatise on Geochemistry, eds. Holland, H. D. \& Turekian, K. K. (Elsevier-Perghamon, Oxford) 201

McKeegan, K. D. 1987, Science, 237, 1468

Messenger, S. 2000, Nature, 404, 968

Molster, F. J. \& Waters, L. B. F. M. 2003, in Astromineralogy, ed. Th. K. Henning (Springer-Verlag Berlin Heidelberg) 121

Mousis, O., Petit, J. -M., Wurm, G., et al. 2007, A\&A, 466, L9

Nakamura, T., Noguchi, T., Tsuchiyama, A., et al. 2008, Science, 321, 1664

Nguyen, A. N., Busemann, H. \& Nittler, L. R. 2007, Lunar Planet. Sci. XXXVIII, 2332 (abstr.) 
Olofsson, J., Augereau, J. -C., van Dishoeck, E. F., et al. 2009, A\&A, 507, 327

Price, M. C., Kearsley, A. T., Burchell, M. J., et al. 2010, MAPS, 45, 1409

Rubin, A. E. \& Keil, K. 1984, Meteoritics, 19, 135

Rubin, A. E. \& Grossman, J. N. 1987, Meteoritics, 22, 237

Sandford, S. A. \& Walker, R. M. 1985, ApJ, 291, 838

Scott, E. R. D. \& Krot A. N. 2003, in Meteorites, Comets and Planets, ed. Davis, A. M., Vol. 1 Treatise on Geochemistry, eds. Holland, H. D. \& Turekian, K. K. (ElsevierPerghamon, Oxford) 143

Shu, F. H., Shang, H., Glassgold, A. E. \& Lee, T. 1997, Science, 277, 1475

Skinner, W. R. \& Leenhouts, J. M. 1993, Lunar Planet. Sci. XXIV, 1315 (abstr.)

Verniani, F. 1969, Space Sci. Rev. 10, 230

Vinković, D. 2009, Nature, 459, 227 
Wirick, S., Flynn, G. J., Keller, L. P., et al. 2009, MAPS, 44, 1611

Zolensky, M. E. 1987, Science, 237, 1466

Zolensky, M. E., Zega, T. J., Yano, H., et al. 2006, Science, 314, 1735

\section{FIGURE LEGENDS}

Figure 1: (A) Secondary electron image of CP IDP U211B6. (B) Darkfield densitythickness contrast image of CP IDP U220GCA dispersed on a thin-film carbon support substrate. The bright grains are mostly pyrrhotite (density $4.7 \mathrm{~g} \mathrm{~cm}^{-3}$ ) and the lighter grains enstatite and forsterite (density $3.2 \mathrm{~g} \mathrm{~cm}^{-3}$ ). Mineral indentifications were verified using quantitative energy-dispersive x-ray spectroscopy. Other indigenous (noncrystalline) constituents include organic carbonaceous matter and amorphous silicates.

Figure 2. Cumulative frequency diagrams of the measured grain size distributions of silicates (green) and sulfides (blue) in CP IDPs U211B6, U220GCA and U212A34A from this study and in Comet 81P/Wild 2 from the systematic survey of Stardust collector foils (Price et al. 2010). Solid lines mark the geometric mean radii whilst shaded areas surrounding represent the standard deviation about each mean (see Table 1). 
Figure 3. Comparison of mean radii of crystalline silicates and sulfides for the CP IDPs U211B6, U220GCA, U212A34A and for Comet 81P/Wild 2. Data is from Table 1 and sources referenced therein. The standard errors of the means are represented by error bars. The grey dashed line represents an error weighted fit to the data, with the equation $\mathrm{M}_{\mathrm{r}}$ sulfide $=0.62 \mathrm{M}_{\mathrm{r}}$ silicate.

Figure 4. Refractory silicate grains in a meteorite from the asteroid belt, a Kuiper Belt comet (81P/Wild 2) and a CP IDP U219C11. (A) Reflected light image of a calciumaluminum inclusion (CAI) in the Allende CV3 meteorite (image: G. MacPherson), (B) backscattered electron image of CAI "Inti" from Comet 81P/Wild 2 (image: H.A. Ishii) and (C) bright field transmission electron image of a polycrystalline aggregate of refractory silicate minerals including anorthite (An) and pyroxene (Рyx) in CP IDP U219C11. 
TABLES

\begin{tabular}{|c|c|c|c|c|}
\hline & $\begin{array}{c}\text { Number } \\
\text { of grains } \\
\text { (n) }\end{array}$ & $\begin{array}{c}\text { Geometric } \\
\text { mean } r \\
(\mathrm{~nm})\end{array}$ & $\begin{array}{c}\text { Standard } \\
\text { deviation in } r \\
(\mathrm{~cm})\end{array}$ & $\begin{array}{l}\text { Standard error of the } \\
\text { mean of } r \\
\left(\mathrm{~g} \mathrm{~cm}^{-2}\right)\end{array}$ \\
\hline \multicolumn{5}{|c|}{ CP IDP U211B6 } \\
\hline Silicates & 497 & 108.90 & $\begin{array}{l}+86.82 \\
-48.30\end{array}$ & $\begin{array}{l}+3.89 \\
-2.17\end{array}$ \\
\hline Sulfides & 1,073 & 65.37 & $\begin{array}{l}+76.72 \\
-35.29\end{array}$ & $\begin{array}{l}+2.34 \\
-1.08\end{array}$ \\
\hline \multicolumn{5}{|c|}{ CPIDP U220GCA } \\
\hline Silicates & 647 & 153.44 & $\begin{array}{l}+137.29 \\
-72.46\end{array}$ & $\begin{array}{l}+5.40 \\
-2.85\end{array}$ \\
\hline Sulfides & 515 & 92.76 & $\begin{array}{l}+113.12 \\
-50.97\end{array}$ & $\begin{array}{l}+4.98 \\
-2.25\end{array}$ \\
\hline \multicolumn{5}{|c|}{ CP IDP U212A34A } \\
\hline Silicates & 322 & 47.31 & $\begin{array}{l}+32.96 \\
-19.42\end{array}$ & $\begin{array}{l}+1.84 \\
-1.08\end{array}$ \\
\hline Sulfides & 115 & 34.01 & $\begin{array}{l}+31.19 \\
-16.27 \\
\end{array}$ & $\begin{array}{l}+2.91 \\
-1.52 \\
\end{array}$ \\
\hline \multicolumn{5}{|c|}{ COMET 81P/WILD 2} \\
\hline Silicates & 66 & 265.96 & $\begin{array}{l}+370.54 \\
-154.83\end{array}$ & $\begin{array}{l}+45.61 \\
-19.06\end{array}$ \\
\hline Sulfides & 44 & 203.03 & $\begin{array}{l}+245.47 \\
-111.12\end{array}$ & $\begin{array}{l}+37.01 \\
-16.75\end{array}$ \\
\hline
\end{tabular}

Table 1. Properties of inner nebula grains in the CP IDPs and comet 81P/Wild 2.

Values for geometric mean radii $(r)$, standard deviations and standard error on the mean for silicate and sulfide crystals in CP IDPs U211B6, U220GCA and U212A34A and Comet 81P/Wild 2. Data for Comet 81P/Wild 2 are derived from Price et al. (2010). 


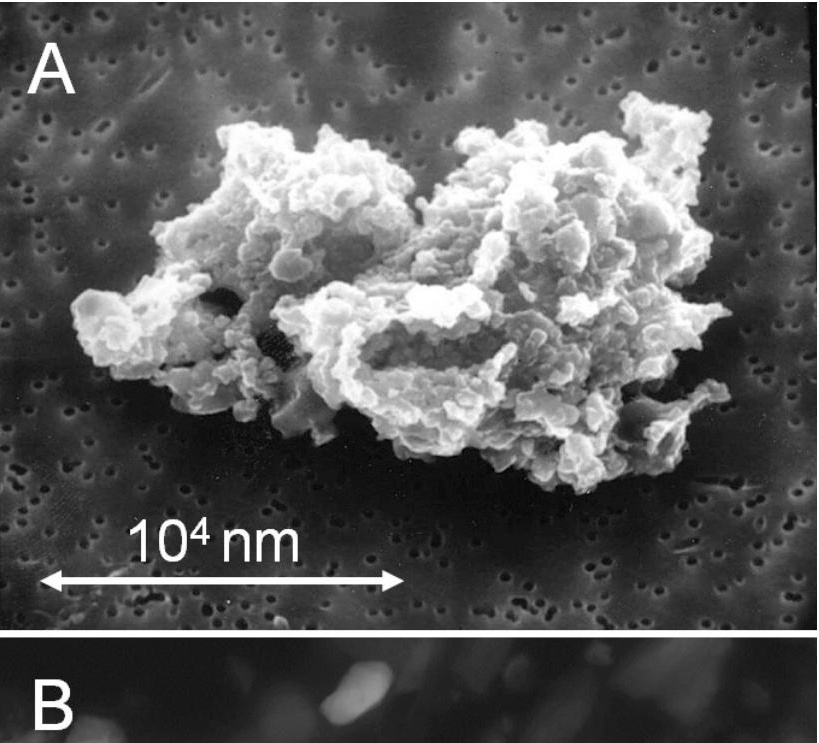



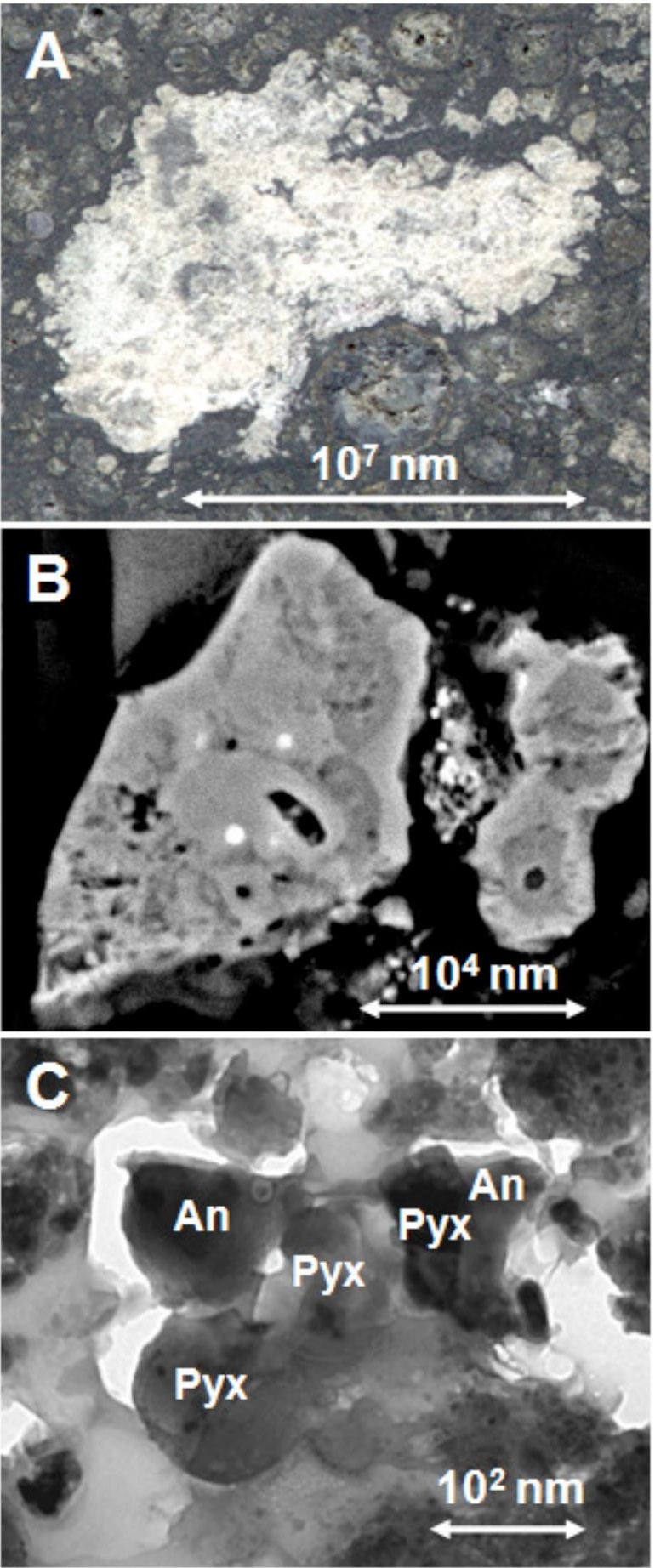